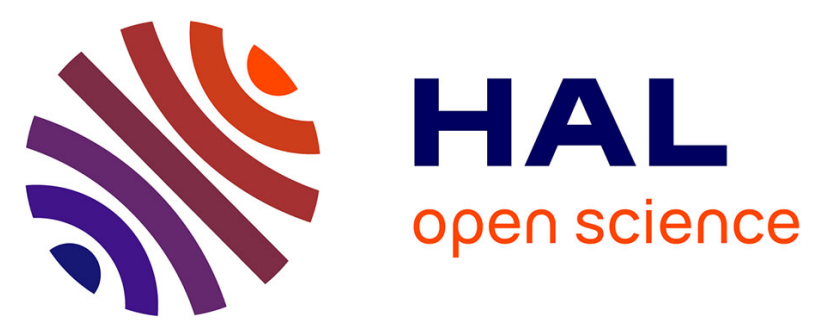

\title{
L'importance attribuée par les usagers des transports publics à l'information fournie par les technologies avancées : un levier pour l'investissement dans des systèmes coopératifs
}

Foteini Mikiki, Maria Morfoulaki, Panagiotis Papaioannou, Georgia

Aifadopoulou

\section{To cite this version:}

Foteini Mikiki, Maria Morfoulaki, Panagiotis Papaioannou, Georgia Aifadopoulou. L'importance attribuée par les usagers des transports publics à l'information fournie par les technologies avancées : un levier pour l'investissement dans des systèmes coopératifs. RTS - Recherche Transports Sécurité, 2013, 2013 (02), pp.125-139. 10.4074/S0761898013002045 . hal-01670619

\section{HAL Id: hal-01670619 \\ https://hal.science/hal-01670619}

Submitted on 21 Dec 2017

HAL is a multi-disciplinary open access archive for the deposit and dissemination of scientific research documents, whether they are published or not. The documents may come from teaching and research institutions in France or abroad, or from public or private research centers.
L'archive ouverte pluridisciplinaire HAL, est destinée au dépôt et à la diffusion de documents scientifiques de niveau recherche, publiés ou non, émanant des établissements d'enseignement et de recherche français ou étrangers, des laboratoires publics ou privés. 


\title{
L’importance attribuée par les usagers des transports publics à l'information fournie par les technologies avancées : un levier pour l'investissement dans des systèmes coopératifs
}

\author{
The importance allocated by public transport users to the information provided by advanced \\ technologies: a lever for investing in co-operative systems
}

\author{
Foteini Mikiki · Maria Morfoulaki · Panagiotis Papaioannou · Georgia Aifadopoulou
}

Reçu le 15 mai 2012 ; accepté le 24 avril 2013

(C) IFSTTAR et Éditions NecPlus 2013

\begin{abstract}
Résumé Le Transport public (TP) doit devenir plus concurrentiel pour lutter contre l'usage croissant de la voiture. Ceci exige de mieux comprendre les besoins des usagers et les déterminants du choix modal. Des systèmes dits coopératifs, permettant une communication en temps réel entre véhicules ou entre ces derniers et l'infrastructure ou l'usager, sont mis en œuvre à travers le monde, améliorant le service et l'information fournis. Améliorer la qualité du service par le biais de la technologie Systèmes de transports intelligents (STI) augmente la satisfaction du client mais demande un investissement durable qui exige suivi et évaluation. Cet article se concentre sur l'application des STI dans Thessalonique (Grèce), où le réseau de bus sera complété d'un réseau de métro en 2015. Ce travail discute le potentiel des systèmes coopératifs du point de vue des exploitants. Au cours de la dernière décennie,
\end{abstract}

\author{
Foteini Mikiki $(\triangle)$ \\ Département d'Ingénierie Civile, Ècole Polytechnique \\ Université Aristote de Thessalonique \\ rue Leonnatou 8, 62122 Serres, Gréce \\ e-mail : fmikiki@yahoo.gr \\ Maria Morfoulaki ( $\square)$ \\ Institut Hellénique des Transports \\ $6^{\mathrm{e}} \mathrm{km}$ rue Charilaou, BP 361 \\ 57001, Thermi, Thessalonique, Grèce \\ e-mail :marmor@certh.gr \\ Panagiotis Papaioannou $(\square)$ \\ Département d'Ingénierie Civile, École Polytechnique, \\ Université Aristote de Thessalonique, \\ 54124 Thessalonique, Grèce \\ e-mail : papa@vergina.eng.auth.gr. \\ Georgia Aifadopoulou $(\bowtie)$ \\ Institut Hellénique des Transports, \\ $6^{\mathrm{e}} \mathrm{km}$ rue Charilaou, BP 361, \\ 57001 Thermi, Thessalonique, Grèce \\ e-mail : gea@certh.gr
}

plusieurs enquêtes ont mesuré la satisfaction des usagers et l'importance attribuée aux applications STI. La clientèle semble porter une appréciation positive et durable sur ces applications et la tendance d'utilisation les transports publics est croissante. La prise en compte du point de vue du client est recommandée aux opérateurs qui peuvent ainsi planifier des améliorations pour répondre aux besoins, guider les choix, et augmenter leur part de marché. Les systèmes coopératifs exigent un investissement considérable et le consensus des acteurs, mais aussi le soutien des clients. Les opérateurs, qui investissent déjà, devront argumenter sur l'importance de telles applications pour les usagers qui en sont de plus en plus demandeurs.

Mots clés transport public · importance de l'information . satisfaction du client $\cdot$ systèmes coopératifs

Abstract Faced with increasing car use, Public Transport (PT) needs to become more competitive. This requires better understanding of both the user needs and the determinants of mode choice. So-called Cooperative Systems, i.e. real-time communications between the vehicles and between them and the infrastructure or the user, are applied worldwide enhancing system performance and information provision which contribute to service quality. Quality improvement based on ITS (Intelligent Transport Systems) help increasing customer satisfaction but need lasting investments that require monitoring and evaluation. This paper focuses on ITS introduction in Thessaloniki, Greece, where PT involves bus services and a metro network by 2015 . The potential of cooperative systems deployment is discussed within the operator's perspective. Several surveys during the last decade recorded users' satisfaction and the importance they grant to ITS applications in Thessaloniki. As a result, users seem to show a positive appreciation of such applications and a propensity to increase their PT use, as 
well. It is recommended that operators take better into account the users' point of view when they plan system development, in order to better fulfill user needs and guide their choices, increasing thus PT share. Cooperative systems deployment requires considerable investment and stakeholders' consensus but also supportive PT customers who ask for advanced mobility services. Thus, PT operators, already investing, should try to convince all parties that such applications are important to users.

Keywords Public transport - Information importance . User satisfaction · Cooperative systems

\section{Le transport public et les applications STI}

Dans une ère où la technologie est employée pour augmenter le nombre de services mis à disposition dans de nombreux domaines, le secteur de transport public ne fait pas exception. Après la révolution des Technologies de l'information et de la communication (TIC), la Télématique avancée des transports s'est développée (ATT - Advanced Transport Telematics) menant au concept des Systèmes de transport intelligents (STI) et des Systèmes avancés d'information des voyageurs (ATIS - Advanced Traveller Information Systems). Depuis plus d'une décennie, des informations peuvent être fournies aux voyageurs à l'aide d'une variété de médias [1].

L'intérêt pour les ATIS a rapidement cru au cours des dernières années, aboutissant déjà à un grand volume de littérature académique en la matière ( $c f$. [2] pour quelques contributions notables). En outre, plusieurs initiatives politiques ont favorisé le déploiement d'ATIS dans des réseaux de transport (par exemple, [3], [4]). Une justification fondamentale de cet intérêt croissant et des investissements conséquents nécessités pour le développement et le déploiement de tels services tient au fait que les réseaux de transports urbains multimodaux deviennent de plus en plus complexes, rendant pratiquement impossible pour les voyageurs une connaissance complète de tous les facteurs qui peuvent influencer leurs choix en matière de déplacement.

À part cette utilité déjà mentionnée face à une structure complexe du service, les applications STI contribuent aussi bien à améliorer la qualité de l'exploitation que la qualité de service perçue ; elles jouent ainsi un rôle positif en termes de marketing et de communication. «Les nouvelles technologies offrent une valeur supplémentaire au client, spécialement psychologiquement, et ont le potentiel de changer son comportement et de contribuer à résoudre des problèmes de mobilité » [5].

À cet égard, les systèmes de transport intelligents ont aussi été l'objet d'importantes activités de recherche dans le contexte de la dynamique comportementale. Les applications et les orientations récentes de la recherche ont déclenché l'intérêt pour d'autres approches en science de gestion et en recherche opérationnelle, particulièrement en Amérique du Nord.

Les grands pays sont engagés dans une course mondiale dans le champ des STI et ont tous des programmes d'investissement dans ce secteur jugé prometteur ${ }^{1}$. Les systèmes intelligents sont nés de la conjonction des révolutions technologiques dans les quatre domaines de l'informatique, des télécommunications numériques, de la géolocalisation des mobiles par satellite et des systèmes automatiques. Le terme générique « système de transports intelligents » vient des États-Unis. Depuis le début des années 1990, différents programmes Intelligent Transport System se sont succédés avec pour perspective initiale la création d'un système autoroutier automatique. Le programme Systèmes de transport public avancés (Advanced Public Transportation Systems APTS) de la Federal Transit Administration dans les années 1990 incluait des projets qui démontraient l'application des technologies de pointe aux systèmes de transport public [6]. Depuis, les recherches et les applications en matière de STI se sont axées non pas vers la robotisation des transports, mais vers la valorisation des Nouvelles technologies de l'information et de la communication (NTIC) ainsi que la mise en place des systèmes automatiques. Les raisons invoquées pour ce changement de priorité sont notamment un contexte peu favorable à la mise en œuvre d'une conduite automatisée à grande échelle [7].

À l'Est, les APTS font partie de la démonstration de la construction des STI dans beaucoup de villes, telles que Pékin, Shanghai, Qingdao, Hangzhou, et Chongqing, dans le $10^{e}$ Programme de science et technologie [8] en tant que sous-ensemble important de systèmes intelligents de transport et élément essentiel pour maîtriser la congestion urbaine et améliorer le niveau de service des transports publics.

En Europe, les opérateurs de Transports publics (TP) emploient aujourd'hui de plus en plus de systèmes intelligents de transport qui facilitent leurs opérations journalières dans plusieurs domaines. Les Systèmes d'information des passagers en temps réel (RTPI) sont très répandus dans les transports publics modernes et des investissements considérables sont consacrés chaque année à de telles applications [9]. Souvent, l'installation de systèmes d'affichage en temps réel aux arrêts est juste une des mesures prises pour améliorer la qualité du service offert aux voyageurs [10]. On admet largement que les RTPI offrent des avantages significatifs aux utilisateurs de TP, existants ou potentiels,

\footnotetext{
${ }^{1}$ Par exemple pour le Canada, voir http://www.tc.gc.ca/ innovation/sti/fra/menu.htm ; pour le Japon, http://www.bulletinselectroniques.com/rapports/smm10_046.htm.
} 
en fournissant une gamme étendue d'informations. À cet égard, les systèmes de RTPI améliorent le service des TP et contribuent à un transfert vers des modes de transport plus durables - en réduisant l'utilisation de voiture et la congestion routière et en améliorant l'efficacité globale du système de transport avec des flux plus aisés et des choix mieux justifiés. Les systèmes de RTPI se fondent simplement sur les technologies d'AVL (Automatic Vehicle Location) qui permettent la localisation spatiale d'un véhicule spécifique et fournissent au public des informations en temps réel qui permettent d'augmenter la satisfaction des passagers en réduisant à la fois le temps réel d'attente et l'effet de perception négative de l'attente en cas de retard [11]. Ces systèmes offrent généralement des informations sur l'heure prévue d'arrivée et de départ, sur la localisation des véhicules, sur le service, sur les perturbations et retards éventuels, sur les tarifs, sur les correspondances et les itinéraires, ainsi que d'autres informations telles que la date et l'heure, la météo, des annonces publiques et des informations liées à la sécurité. Ces informations fournies aux passagers les aident à la prise de décisions avant ou pendant le voyage. L'accès à cette information avant le voyage peut aider à diminuer le temps d'attente réel et, quand l'information est disponible en route, elle peut aider à réduire le temps d'attente perçu [12]. La fourniture d'aides de RTPI améliore l'image des entreprises de TP au sein de leurs communautés ([13], [14], [15]) et beaucoup d'études concluent que les systèmes de RTPI offrent plusieurs avantages aux utilisateurs de TP et que la majorité d'entre-eux en sont assez satisfaits ([16], [17], [18], [19]).

Comme déjà mentionné, les opérateurs de TP sont face à un éventail de pressions d'un marché concurrentiel et moins régulé [20]. Soit les opérateurs répondent à ces pressions en apportant des améliorations (en assurant ainsi leur viabilité ou en renforçant leur position), soit ils restent passifs (ce qui exige une intervention plus forte ultérieurement ou les conduit à l'échec). En outre, beaucoup d'opérateurs et d'autorités de TP utilisent la satisfaction du client comme une mesure de contrôle de la qualité.

Toute proposition d'amélioration, y compris les applications STI, devrait être évaluée avec soin, en ayant à l'esprit que l'utilisateur devrait être au cœur de n'importe quelle mesure et que ce sont ses perceptions et ses préférences qui doivent guider les options : «Étant donné la liaison entre la perception de la qualité de service et le nombre de voyageurs, la future recherche sur les nouvelles technologies de TP devrait se focaliser sur celles qui ont le plus grand potentiel d'augmentation du nombre de voyageurs » [21]. L'investissement global dans un système STI sophistiqué pour les TP (conception, mise en place et exploitation), offrant une gamme de fonctions élargie, n'est pas négligeable comparé aux coûts totaux d'exploitation des TP [22]. À propos des coûts et dans un effort pour suivre les développements technologiques et en tirer le meilleur parti, les exploitants doivent en permanence envisager d'investir plus en amont dans des systèmes plus sophistiqués - dont les systèmes coopératifs qui se profilent comme étant l'avenir des STI. Une manière d'étudier la pertinence d'investissements supplémentaires dans ce domaine est de mesurer l'acceptation par les utilisateurs, l'évolution de celle-ci au fil du temps et l'importance qu'ils accordent aux technologies, mais aussi de faire participer tous les acteurs compétents pour anticiper la sophistication croissante des applications qui intègrent des composantes coopératives.

\section{Systèmes coopératifs : l'avenir de STI dans le secteur du transport public}

Les systèmes coopératifs constituent une extension des applications STI existantes, permettant la communication entre véhicules (V2V - vehicle to vehicle) ou avec l'infrastructure (V2I - vehicle to infrastructure ou I2V) et dont le but ultime est d'améliorer la gestion du trafic et la sécurité. Comme déjà évoqué, la communication des systèmes télématiques avec un usager possédant un téléphone portable intelligent ou tout autre dispositif lui permettant d'acquérir des informations en temps réel et de faire ses propres choix de déplacement peut aussi être considérée comme un système coopératif dans lequel la notion d'usager intelligent est incorporée. Les téléphones mobiles sont appelés à jouer un rôle clé dans ces dispositifs de mobilité, en fournissant aux usagers des informations personnalisées et à tout moment. Des applications sortent régulièrement pour choisir et réserver un véhicule, calculer l'empreinte carbone d'un déplacement, connaître sa vitesse en temps réel, etc. Une réflexion est également produite sur ce qui est appelé l'Assistant personnel de mobilité (APM), basé sur les technologies du GPS, de la téléphonie mobile et d'internet. Il devrait fournir à l'usager toutes les informations dont il a besoin en temps réel pour choisir les meilleures modalités de déplacement sur la base de critères qu'il aura lui-même choisis (coût, temps, pollution, lien social), tout en apportant à l'autorité organisatrice des transports une vision globale sur la mobilité réelle [23]. Les systèmes coopératifs peuvent contribuer à une meilleure gestion des transports urbains de plusieurs manières. Un avantage potentiel des systèmes coopératifs, important pour une autorité locale, est la qualité et la quantité de données en temps réel recueillies par les véhicules qui en sont équipés. D'autres avantages offerts par cette technologie comprennent : une gestion améliorée de la voirie, une plus grande efficacité des systèmes de transport en commun, des émissions réduites, une sécurité routière améliorée pour tous les utilisateurs de la route, une congestion réduite et une réponse améliorée et plus efficace face aux risques et 
aux incidents. Il est généralement admis que les systèmes coopératifs peuvent apporter des avantages en matière de politique locale [24].

Les systèmes coopératifs constituent un secteur en expansion où sont développées des technologies d'avantgarde dont les avantages ont été démontrés à l'échelle mondiale. Ces avantages, déjà présentés brièvement, sont actuellement le sujet de travail de plusieurs dépositaires, fabricants de voitures et fournisseurs de solutions STI. De grands projets européens de recherche et développement tels que CVIS, Safespot, Satie, COMeSafety2, Instant Mobility etc., ou projets pilotes tels que Freilot et des Essais opérationnels sur le terrain (Field Operational Tests - FOTs) se concentrent sur le déploiement de systèmes coopératifs. Ils sont explicitement mentionnés dans le plan d'action STI (Action 4 - Intégration du véhicule dans l'infrastructure de transport) [25]. Des États-membres se dirigent vers les systèmes coopératifs, l'exemple notable étant celui des Pays-Bas qui ont développé une feuille de route nationale pour leur déploiement. En dépit de ce courant, le déploiement peut être bloqué si les autorités urbaines et régionales ne sont pas activement engagées dans le processus. Il est clair que ce genre de systèmes dépasse le domaine de responsabilité d'un opérateur de TP et la participation de différents acteurs fait de l'acceptation d'un tel investissement une question cruciale pour la communauté. C'est pourquoi l'opérateur qui a déjà mis sur pied des applications STI peut s'appuyer sur la satisfaction du client pour convaincre d'autres acteurs et les autorités de s'engager dans des réalisations plus ambitieuses. Les autorités locales ont un rôle important à jouer dans le déploiement de systèmes coopératifs pour ce qui concerne les Équipements au sol (Road Side Units - RSUs). L'installation de ces équipements et leur entretien sont essentiels au fonctionnement des systèmes coopératifs de V2I et d'I2V pour n'importe quel type d'application, tout comme les informations sur l'état du réseau routier ou la gestion des incidents [26].

Enfin et surtout, la question d'acceptation par les utilisateurs est celle sur laquelle ce travail se focalise. Puisque de nombreux acteurs doivent être impliqués dans le déploiement des systèmes coopératifs, la question de l'acceptation par les utilisateurs doit être mieux comprise. Un niveau élevé de satisfaction des utilisateurs et un public familiarisé avec les nouvelles technologies et leurs futurs ajustements peuvent amplifier le soutien social et créer un niveau de demande propice à leur déploiement. L'acceptation par les utilisateurs est également nécessaire en matière de données personnelles (notamment les données de localisation). Des règles claires et des procédures de gestion de ces questions doivent être en place pour que les autorités locales puissent déployer les systèmes coopératifs à grande échelle.
En résumé, pour un déploiement accéléré des systèmes coopératifs les opérateurs des TP devraient «vendre » l'idée aux autorités locales d'une manière concrète. Ces dernières ont un rôle important à jouer en particulier en ce qui concerne l'installation et la maintenance des équipements au sol. Les autorités locales seront intéressées à déployer les systèmes coopératifs s'ils peuvent offrir des avantages significatifs en permettant d'atteindre des objectifs politiques, notamment la circulation efficace des personnes et des marchandises - indépendamment du mode de transport.

Cet article discute l'hypothèse que les applications des STI sont des composants essentiels de la qualité de service des TP et que les systèmes coopératifs promettent une future mobilité urbaine considérablement facilitée. Par ailleurs, de tels systèmes exigent un investissement important à long terme afin de fournir un éventail de services de qualité. Après une présentation brève de l'histoire des applications STI dans le domaine des TP et de leur futur potentiel enrichi par des systèmes coopératifs, l'article se focalise sur l'acceptation des systèmes existants par l'usager et détaille le cas de la ville de Thessalonique (Grèce), où la mise en œuvre des STI prévoit une coopération entre le système de télématique et l'usager. Dans une première phase à Thessalonique, seules des applications conventionnelles de STI ont été mises en place. Le réseau de TP de cette ville est composé de lignes de bus, auxquelles viendra s'ajouter un métro souterrain en 2015. En partant d'une enquête ayant évalué le niveau de satisfaction des usagers avant la mise en place de STI, le présent travail analyse l'évolution de l'appréciation du public sur cette dernière décennie - jalonnée par trois efforts de recherche importants visant à capter le point de vue des usagers - : en 2003, en l'absence de toute implémentation de STI, en 2007 où la mise en œuvre de la télématique a démarré et, plus récemment, en 2010 avec un système complètement opérationnel. Une analyse bibliographique est présentée sur la relation entre la fourniture de l'information aux usagers des TP et l'acceptation de ces derniers. Puis, après avoir présenté le cas de Thessalonique, l'article détaille l'approche méthodologique adoptée et les résultats obtenus. Enfin, l'article porte sur les conclusions qui peuvent être dégagées sur la relation entre l'acceptation par les usagers et le niveau d'investissement en STI en fonction de l'importance que les usagers lui accordent, ce qui peut ouvrir le champ à un futur déploiement de systèmes coopératifs. On peut effectivement pressentir un potentiel pour accroître la qualité des services, potentiel suffisamment important pour justifier d'y consacrer des ressources supplémentaires en vue d'augmenter la satisfaction des usagers et un accroissement de l'usage des TP. La discussion se concentre sur les contributions de la présente étude et sur des recommandations à l'adresse des TP et des décideurs. 


\section{Information fournie aux usagers des TP et son acceptation}

Afin de maintenir le niveau actuel de leur clientèle et d'en attirer une nouvelle, les TP visent à assurer la satisfaction de leurs utilisateurs par la création de nouveaux et de meilleurs services. La manière dont la satisfaction des utilisateurs est liée à la qualité du service est une préoccupation mais également un élément essentiel en matière de politique d'entreprise des TP, car sa connaissance permet d'adopter des choix plus pertinents et mieux adaptés aux besoins des utilisateurs. Ceci justifie le besoin de pouvoir disposer d'un mécanisme d'évaluation et de mesure du degré de satisfaction des aspirations des utilisateurs et de leur satisfaction : un Index de satisfaction du client (Customer Satisfaction Index - CSI). « L'autorité organisatrice des TP peut ainsi déterminer les mesures spécifiques qui doivent être prises, en vue d'améliorer la satisfaction des usagers » [27].

Les recherches sur la satisfaction de l'usager suggèrent que le déroulement du déplacement et la qualité du service entraînent un effet direct [28] : «La qualité du service des TP reflète la perception qu'a le passager du déroulement du déplacement » [29]. Le lien entre la qualité de service et la satisfaction de l'usager a été exploré par plusieurs travaux de recherche [30], [31], [32], [33]. Ces recherches offrent des éclairages importants sur cette relation d'interdépendance, objet de la préoccupation non seulement du monde scientifique du transport, mais aussi des exploitants. Dans ce contexte, il convient de relever l'importance de l'apport des TIC dans l'amélioration du service.

L'information aux voyageurs est un des déterminants de la qualité de service et apporte des bénéfices tant aux utilisateurs qu'à l'exploitant. À cet égard, des STI sont utilisés dans le monde entier afin de répondre à ces attentes et pour atteindre les objectifs exigeants que s'imposent les opérateurs de transport. Les applications basées sur les technologies de l'information semblent également être appréciées des clients [10], mais les effets de celles-ci sur le comportement des clients actuels ou potentiels [34] sont peu connus, comme par exemple sur la « disposition à payer » (willingness to pay) des passagers pour obtenir de l'information en temps réel aux arrêts et à bord des véhicules [35] ou sur l'effet de l'information sur la demande.

Un effort de recherche important a été mené vers le milieu des années 1990 sur les modifications des comportements de déplacement résultant des systèmes d'information aux voyageurs destinés aux conducteurs de voitures [36]. Toutefois, des travaux de cette nature portant sur les TP sont plus limités et nos connaissances sur l'effet de l'information en temps réel fournie aux usagers des TP sont lacunaires [37], [38], [39]. Au moment des études, il n'y avait pas d'éléments précis quant à une augmentation de l'usage des $\mathrm{TP}$ en réponse à une mise à disposition de l'information en temps réel. Une augmentation de l'utilisation des TP observée après l'introduction de STI [40] aurait pu être assimilée à une modification de comportement ; toutefois, la moitié des personnes interrogées n'a pas considéré que l'information constituait un service supplémentaire susceptible d'augmenter son niveau de satisfaction et de conduire à un changement de son comportement [41]. Selon Kronborg et al., la mise à disposition de l'information en temps réel accroît bien l'utilisation des TP, mais cet effet est difficile à quantifier [42]. Des travaux de recherche montraient clairement que les usagers apprécient l'information en temps réel et se sentent plus à l'aise lorsqu'ils en bénéficient [37].

Ultérieurement, de tels systèmes ont été mis en place à large échelle et les efforts d'évaluation de leur efficacité ont été multipliés. Traditionnellement, les exploitants des TP mènent des enquêtes de satisfaction de la clientèle afin d'évaluer leur possibilité d'améliorer le service. Durant une décennie, l'émergence des STI a suscité des activités de recherche y compris dans le domaine de la dynamique comportementale et des travaux récents suggèrent l'existence d'effets psychologiques chez les usagers [43] et que "l'utilité potentielle de telles technologies, du point de vue de l'usager, est leur capacité d'augmenter la qualité perçue du service» [21]. Aujourd'hui, notre connaissance est plus complète en ce qui concerne l'acceptabilité par les usagers des TP qui, bien que sensibles au prix, acceptent de payer plus si l'information, telle que celle fournie en temps réel, représente une plus-value [44].

Au sein du monde des TP, un consensus généralisé semble exister selon lequel l'information en temps réel augmente le niveau de satisfaction de la clientèle et représente un potentiel intéressant en vue d'accroître l'usage des TP. L'information aux voyageurs en temps réel peut avoir un effet compensatoire en réduisant les temps d'attente et l'incertitude qui leur est liée en cas de transbordements. On perçoit ainsi une possibilité que de tels systèmes soient de nature à compenser les déficiences perçues du service, améliorant ainsi la satisfaction de la clientèle et augmentant l'utilisation des TP. Des questions restent cependant ouvertes concernant cette chaîne : présence de STI - amélioration de la qualité de service - augmentation de la satisfaction de la clientèle - modification du comportement - augmentation de l'utilisation.

Une question cruciale est celle de la différence d'acceptation entre utilisateurs et non-utilisateurs des TP. Les études existantes sur le potentiel des systèmes d'information d'attirer plus d'usagers, et plus spécialement parmi les non-utilisateurs, restent peu concluantes. Une étude d'Abdel-Aty indique que l'information présente un 
potentiel de croissance de l'usage des TP auprès des non-utilisateurs pour les déplacements pendulaires [39]. Selon un panel d'experts [34], des effets ont été observés sur des utilisateurs expérimentés et occasionnels qui incluaient les TP dans leur ensemble de choix possibles. Pour les usagers expérimentés, l'information a permis d'opter pour des itinéraires plus faciles et plus rapides. Pour les occasionnels, le choix des options a été amélioré, ce qui leur a aussi permis d'opter pour des itinéraires plus efficaces. De surcroît, une information efficace et ciblée à l'adresse des occasionnels, permet d'abaisser le « seuil d'entrée au système » et les encourage à se montrer plus inventifs. L'information a été grandement appréciée et a aidé ces usagers à se sentir plus « en contrôle». Si une expérience se montre positive, ceci conduit à la répéter, ce qui augmente l'utilisation des TP. Dziekan conclut dans sa revue que l'information a été grandement appréciée des usagers et les a aidés à se sentir plus en contrôle tout en améliorant globalement la qualité de leurs déplacements [34]. Un rapport américain indique que, grâce à l'information avant le voyage, le confort du voyageur est accru car l'incertitude et l'anxiété sont réduites, aussi lorsque des emplacements existent consacrés aux annonces pour le public [45]. L'utilisation des TP devrait augmenter (tant pour les usagers actuels que par l'addition de nouveaux usagers) car les TP deviennent plus attractifs et plus faciles d'utilisation, comme démontré par plusieurs exemples : Helsinki, Bruxelles, Liverpool ou Southampton. Le même travail présente quelques exemples quantifiés d'augmentation de la satisfaction de la clientèle à Helsinki, Londres, Turin et dans le Connecticut. Les systèmes embarqués, n'ont pas fait l'objet d'exemples quantifiés documentés, mais une augmentation peut être attendue grâce à un confort accru. Ce travail montre également l'évaluation quantitative d'une attente d'utilisation accrue grâce à la Localisation automatique des véhicules (AVL) à Toronto, Denver et Milwaukee. Dans ces régions, une meilleure satisfaction de la clientèle est signalée, liée à une meilleure fiabilité du service facilitant l'information aux passagers, y compris la connaissance de la localisation du bus qui réduit aussi l'anxiété. A contrario, Chorus et al. montrent que l'impact de l'information TP sur les conducteurs de voiture reste très limité, même si cette information est très favorable aux $\mathrm{TP}$ en général et suggèrent ainsi de rester modéré quant à l'estimation du potentiel de transfert modal [46]. Dans une tentative de quantification du rendement de l'investissement en systèmes d'information sur l'heure d'arrivée des bus, Cham et al. n'incluent pas le potentiel d'augmentation de l'utilisation des TP parmi les bénéfices, car leurs résultats n'offrent pas une base suffisante pour ce faire [22].

La bibliographie donne une image mitigée. D'un côté, les études de préférences déclarées et les simulations montrent une corrélation positive entre l'information et le transfert modal. De l'autre, les expériences réelles n'apportent pas de preuve définitive d'utilisation accrue des TP grâce à une meilleure information, bien que des réponses psychologiques positives puissent généralement être observées. Ces résultats incitent à une prudence qui devrait être de mise lorsqu'il s'agit de prévoir l'effet du déploiement de systèmes d'information en temps réel sur l'accroissement de l'utilisation des TP. Une enquête de préférences déclarées à Chicago montre que $67 \%$ des répondants auraient augmenté leur utilisation des TP s'ils disposaient de l'information en temps réel, à raison de $60 \%$ pour les usagers actuels et de $70 \%$ pour les non-utilisateurs [47]. À la lumière de ce qui précède, les résultats doivent être considérés avec une certaine réserve [48].

L'information requise n'est normalement pas nécessaire pour les déplacements fréquents sur un itinéraire habituel. Toutefois, dès qu'il s'agit d'itinéraires inconnus ou peu pratiqués, ce qui est plus souvent le cas pour les déplacements liés aux loisirs, l'information sur des appareils mobiles est utile. Les pendulaires ainsi que les voyageurs fréquents apprécient grandement de pouvoir disposer, en cas de perturbation, de l'information sur leurs appareils mobiles. Les enquêtes sur la réponse de la clientèle aux dispositifs d'affichage électronique aux arrêts - dans de nombreuses villes européennes, telles que Bruxelles ou Glasgow, menées dans le cadre du projet Infopolis2 montrent une réponse globale très positive [34]. Cependant, l'apparition des STI a déclenché des activités de recherche dans le contexte de la dynamique comportementale et les travaux récents suggèrent des effets psychologiques sur les utilisateurs [43] ; " l'utilité potentielle de telles technologies du point de vue des consommateurs est sa capacité d'améliorer la qualité perçue du service » [21].

Bien que les avantages de STI semblent tangibles, il est parfois difficile de les évaluer en termes monétaires et il est souvent difficile de convaincre les décideurs de la praticabilité d'un tel système ou de celle de systèmes plus coopératifs. Cependant une chose est certaine, une fois qu'un tel système est en place personne ne peut penser à l'exploitation de TP sans STI. L'évaluation des STI est un processus complexe en plusieurs pans couvrant différents aspects de ceux-ci : économique et financier, technique, acceptation par les utilisateurs, opérationnel et organisationnel. Un niveau élevé d'acceptation et un niveau élevé de satisfaction de l'utilisateur doivent être atteints dans tous les cas pour qu'un système soit réussi [11]. Le présent travail contribue aux recherches déjà existantes en présentant une étude en trois phases portant sur les usages réguliers avec des conclusions quant aux systèmes en temps réels préférés et l'appréciation que les usagers font de ces services. L'importance que les usagers attribuent aux applications courantes pourrait dicter le rythme d'introduction futur de systèmes coopératifs. 


\section{L'étude de cas de Thessalonique, Grèce}

Le domaine d'étude inclut la région urbaine et périurbaine de la ville de Thessalonique, également connue comme la Région métropolitaine de Thessalonique (RMT). Thessalonique est située dans la région de Macédoine centrale, en Grèce du Nord et couvre une surface de $235 \mathrm{~km}^{2}$. C'est la deuxième ville du pays, avec une population d'approximativement 1000000 habitants dont 380000 actifs. La RMT comprend la ville de Thessalonique et 17 autres municipalités. Le réseau routier est considérablement congestionné, particulièrement au centre de la ville pendant les heures de pointe, conduisant à des retards importants. Approximativement, 2200000 voyages de personnes sont effectués dans la RMT un jour typique de travail (évaluations de 2010). Le centre de la ville en étant l'origine ou la destination (ou les deux), il est intensément pénalisé par le trafic et les problèmes associés (retards, files d'attente, consommation d'énergie excessive et émission des polluants [49]).

Le système de TP dans Thessalonique inclut des lignes de bus et est actuellement exclusivement exploité par l'Organisme des transports urbains de Thessalonique (OASTH) qui agit dans le cadre d'un contrat de concession avec le gouvernement grec. L'exploitation des transports en commun est régulée et surveillée par l'Autorité des transports de Thessalonique, sous la juridiction du Ministère du transport. L'OASTH exploite les 78 lignes de bus de son réseau avec un parc de 622 véhicules. Le nombre annuel de passagers transportés par OASTH est approximativement de 180000000 [50]. Le système de TP sera enrichi par un réseau de métro souterrain, mis en construction en 2006. Le métro coûtera approximativement 1 milliard d'euros et sera prêt d'ici 2015.

La part de marché de TP ne cessait de se réduire depuis le début des années 1990, en raison de la congestion et d'un taux de motorisation croissant ; celui-ci a presque doublé depuis 1988 [11]. L'OASTH qui a pris plusieurs mesures pour maintenir son niveau de clientèle y est parvenu dans l'absolu, mais pas en termes relatifs en raison de l'augmentation de la mobilité personnelle. La réalisation de plusieurs couloirs de bus et la restructuration partielle des lignes du réseau des bus y ont contribué. Cependant, les passagers sont devenus plus exigeants au fil des ans et leur attente en terme de qualité de service a changé. En 2007-2008, afin d'améliorer les services de TP, l'OASTH a mis en œuvre un système d'information aux voyageurs en temps réel, basé sur un système d'AVL précédemment installé. Le budget total de l'AVL et du système de RTPI était approximativement de 4 millions d'euros. L'investissement a inclus l'installation des unités d'Affichage à messages variables (VMS) à l'intérieur des 600 bus de l'opérateur (présentant des messages en grec et en anglais) et 200 systèmes d'affichage intelligents aux arrêts de bus (qui affichent le temps d'attente jusqu'à l'arrivée des trois à dix prochains bus). En plus, l'OASTH a financé l'installation à l'intérieur des véhicules de messages sonores annonçant le prochain arrêt [16].

Le système entier est basé sur des dispositifs embarqués suivant la progression des véhicules et utiles à la gestion du trafic des bus. Recourant aux technologies GPS et GSM, les informations nécessaires, telles que les coordonnées spatiales, le temps global et la vitesse de chaque autobus, sont envoyées à un Centre de gestion du trafic (COT). Tenant compte du trafic routier sur les itinéraires d'autobus, le temps probable pour atteindre les prochains arrêts est calculé et l'information est envoyée à chaque arrêt par GSM.

Une enquête de passagers de TP dans la région de Thessalonique a démontré l'influence forte des applications STI sur le nombre de voyageurs [16]. La majorité de répondants (environ $75 \%$ ) a déclaré leur volonté d'employer le système de TP davantage si une application STI pour l'information et/ou la sûreté des passagers était adoptée. Ce pourcentage correspond à l'intention de répondants d'employer le nouveau système, mais ne renseigne pas sur le comportement réel des utilisateurs. Les enquêtes de préférences déclarées, en combinaison avec l'analyse effective du comportement réel, représentent un outil prometteur pour évaluer les choix d'un utilisateur (potentiel). L'avantage principal de cette méthode est que la priorité de chaque utilisateur est calculée discrètement en tenant compte de la maximisation de son utilité [51]. L'acceptation partielle du système ( $75 \%$ au lieu de $100 \%)$ est une conclusion commune de ces analyses puisque, d'une part, l'utilisateur est au courant des moyens alternatifs d'information déjà existants et, d'autre part, les répondants ont des difficultés à percevoir entièrement les avantages d'un nouveau système qui n'est présenté que par une description courte dans le guide d'entretien.

L'enquête susmentionnée des passagers TP dans la région de Thessalonique a également mis en évidence une grande influence des applications STI coopératives sur l'indice de satisfaction des clients. Plus précisément, il s'avère que si le niveau de satisfaction lié à l'information en temps réel aux arrêts d'autobus (ce qui suppose une application STI de localisation automatique des véhicules et un VMS ou un autre affichage électronique aux arrêts informant de l'arrivée du bus suivant) augmente de $30 \%$, c'est-à-dire de $6,0 \%$ à $7,8 \%$, alors la satisfaction globale, exprimée par l'indice de satisfaction des clients, passe de $6,2 \%$ à $6,7 \%$ ou à $8 \%[16]$.

L'évaluation positive du système existant de RTPI et les niveaux assez élevés de satisfaction de la teneur et de la fiabilité de l'information fournie ont été également attestés par un travail entrepris en Thessalonique et qui a enregistré le point de vue des utilisateurs en 2008 [11]. 
Le travail actuel a essayé d'entreprendre une autre évaluation des préférences des utilisateurs et de l'augmentation potentielle du nombre de voyageurs après une période de pleine opération des STI mis en application. Les hypothèses faites étaient que l'intérêt continu de l'utilisateur au cours des années pour les services d'information pourrait permettre de considérer le déploiement de plus d'applications STI comme celles, plus avancées, nommées coopératives.

Dans la ville de Thessalonique (Grèce), les décisions d'investissements dans les STI pour le transport public relevaient jusqu'ici de la responsabilité de l'opérateur des services de bus de la région métropolitaine de Thessalonique. Pour passer à des systèmes plus avancés, l'implication d'autres acteurs et de décideurs est indispensable, et il paraît logique que ceux-ci aient besoin de connaître le point de vue des utilisateurs et leur niveau d'acceptation pour envisager des investissements rentables. L'éclairage fourni par les priorités des usagers peut guider de futures réalisations en vue d'améliorer la qualité de service à l'aide de systèmes coopératifs.

\section{Méthodologie}

Étant donné le consensus général sur la fourniture d'information en temps réel comme une composante de la qualité de service, le présent travail vise à étudier l'importance attribuée à de tels services en théorie et dans la réalité (après la mise en œuvre). En outre, il analyse les préférences des utilisateurs potentiels en matière de diffusion de l'information. L'hypothèse est que les résultats pourraient orienter les décisions des opérateurs qui devraient correspondre aux besoins et aux priorités des utilisateurs, comme le suggère la littérature. L'analyse présentée a également été façonnée par les limites imposées par les données disponibles. Enfin, dans une vision prospective de déploiement de systèmes coopératifs, l'aspect coopératif étudié est celui des systèmes avec l'utilisateur.

Dans ce travail, on considère l'information comme le pivot des systèmes de transport intelligents. Elle met en lumière les conditions par rapport auxquelles on peut agir, ainsi que les tendances qui servent à la planification du système. L'information aux voyageurs amène ces derniers à prendre des décisions. Notre approche porte alors sur l'importance attribuée par les utilisateurs eux-mêmes comme un indice d'appréciation - sur des technologies avancées avec lesquelles le public coopère.

L'évaluation des technologies coopératives de transport en commun mises en application jusqu'ici (telles que les systèmes d'information en temps réel avant et pendant le voyage) a été réalisée en trois étapes en utilisant des résultats d'enquêtes.
La première étape est basée sur les résultats d'une enquête de satisfaction des clients réalisée dans le cadre d'une thèse de doctorat rédigée en 2003 [52] - c'est l'enquête qui couvre la période avant l'application. L'évaluation de la qualité de service a été rendue possible en mesurant le niveau de satisfaction à l'aide d'un certain nombre d'attributs de service (paramètres). Pour chaque attribut, les répondants ont été invités à noter l'importance et la satisfaction (gamme des valeurs 1-10). Les répondants ont été séparés en deux catégories principales : 219 utilisateurs fréquents et 162 ne l'étant pas.

Les données de la deuxième étape proviennent des résultats d'une enquête de clientèle de TP qui a eu lieu après la mise en service pilote du système, en 2007, afin d'estimer la première impression des passagers quant à l'utilisation des systèmes STI. Les objectifs du questionnaire étaient de mesurer le niveau de la satisfaction des répondants, d'identifier et d'apprécier les impacts des applications STI. Les répondants ont été choisis sur une base aléatoire à différents endroits dans la région d'étude. Un échantillon total de 223 questionnaires valides a été recueilli, correspondant à 164 utilisateurs fréquents de TP et 59 non-fréquents.

Finalement, la troisième étape a eu lieu dans Thessalonique en 2010 dans le cadre du projet Intelligent urban mobility management and traffic control system for the improvement of the urban environment quality in the central area of Thessaloniki's agglomeration afin d'étudier les services fournis par un centre de mobilité que les citoyens considèrent comme le plus utile pour eux. Un questionnaire destiné à 5000 utilisateurs et non-utilisateurs des transports en commun a permis d'évaluer les technologies STI existantes et d'identifier leurs préférences pour de nouveaux services intelligents. Les réponses des 1500 utilisateurs captifs de TP concernant l'importance des systèmes d'information en temps réel existants de l'OASTH ont été employées pour cette analyse.

Les trois enquêtes n'étaient pas conçues à l'identique et ne visaient pas le même but ; il a donc fallu les homogénéiser et mettre leurs résultats en commun. Les deux premières enquêtes visaient à évaluer l'importance et la satisfaction des passagers quant à des mesures et à des actions spécifiques. À cette fin, un échantillon de 500 réponses a été considéré comme satisfaisant. Par contre, l'échantillon de la troisième enquête était plus grand, car celle-ci visait à établir une matrice O-D (origine-destination) pour les déplacements quotidiens à Thessalonique.

La perception de l'importance résulte des préférences personnelles préexistantes, de l'expérience et de la satisfaction des usagers. C'est un indice intéressant de l'attrait d'un composant de la qualité de service, comme le déploiement des STI, et pourrait guider de futures décisions. Les utilisateurs réguliers des TP, qu'ils soient captifs ou non, ont 


\begin{tabular}{|ll|}
\hline Tableau 1 Échantillon des trois étapes d'étude & \\
\hline Période & Échantillon \\
\hline Avant l'application & 219 \\
Application pilote & 164 \\
Application complète & 1500 \\
\hline
\end{tabular}

de par leur expérience une vision plus claire des services offerts et de leurs valeurs relatives. Comme ils utilisent les TP au moins deux fois par jour, ils ont une opinion ferme sur à la manière dont ces technologies et systèmes coopératifs influent sur la qualité du service et sur leur vie quotidienne (les STI, par exemple, peuvent réduire leur temps d'attente et, par conséquent, leur temps de voyage). C'est pour cette raison que le présent travail se base sur les réactions des utilisateurs réguliers. L'échantillonnage utilisé pour les trois phases de l'analyse est présenté ci-dessous.

L'évolution dans le temps de la perception qu'ont les utilisateurs de l'importance des systèmes d'information avant et pendant le voyage est analysée dans le prochain chapitre.

\section{Analyse des données et résultats}

Étant donné que les différentes enquêtes ne suivaient pas la même approche, un effort a été fait pour décrire les attributs communs dans les trois cas. Ce qui semble être comparable au cours des années est l'importance attribuée à la présence des STI et à leur contribution à la qualité de service.

En 2003, l'information fournie par l'autorité de transport en commun était limitée à des feuillets d'information d'horaires des TP, de tarifs, etc. À ce stade, cette information historique et statique n'était pas en mesure d'affecter la décision finale du citoyen d'utiliser ou pas le TP. Les citoyens n'étaient pas au courant de l'utilisation d'un système d'information en temps réel qui pouvait « coopérer » avec eux. La figure 1 se rapporte à cette période, fournissant la distribution des répondants selon l'importance qu'ils attribuent à l'existence de l'information en temps réel, avant et pendant le voyage. Les données ci-dessus indiquent que presque $63 \%$ des répondants trouvent ces systèmes coopératifs d'importance élevée, $26 \%$ croient qu'ils sont assez importants et $11 \%$ ne considèrent pas ces systèmes si nécessaires.

La perception par les passagers de l'importance de ces technologies pour l'année 2007, quelques mois après l'implémentation pilote des systèmes d'information en temps réel par l'OASTH, est présentée ci-dessous (Figure 2). Les données disponibles indiquent que presque $98 \%$ des

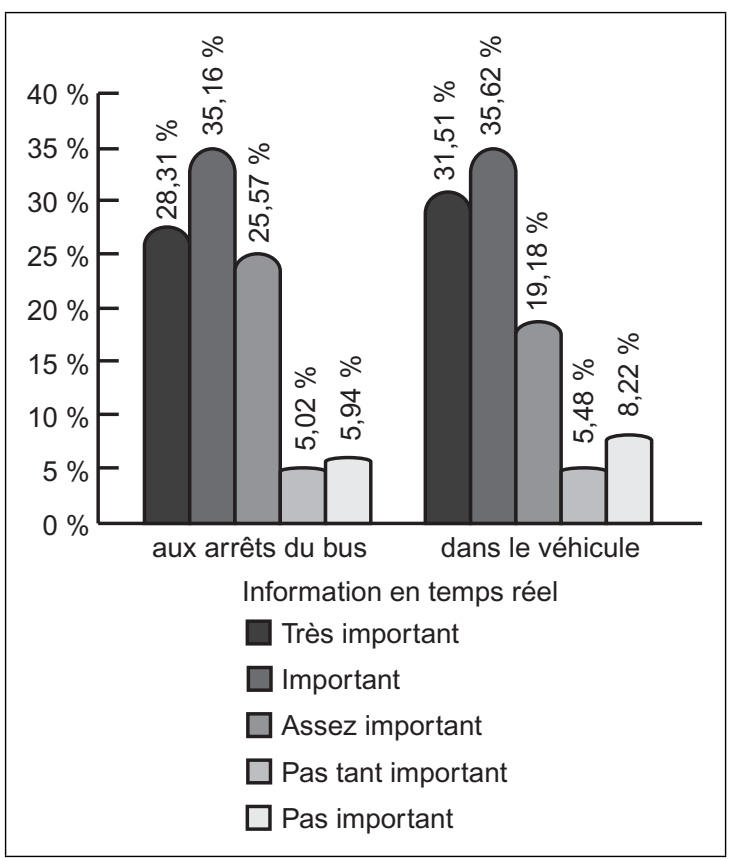

Figure 1 Importance de l'information en temps réel avant l'application

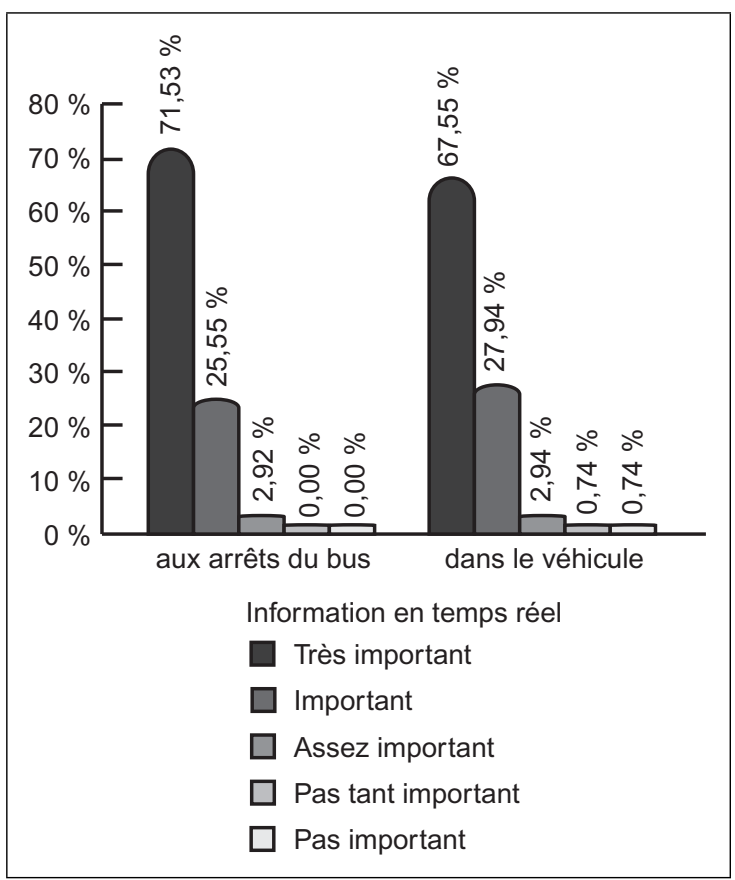

Figure 2 Importance de l'information en temps réel pendant l'application pilote

répondants trouvent ces systèmes d'importance élevée et $2 \%$ croient qu'ils sont assez importants.

Trois ans après la mise en service des systèmes d'information en temps réel par l'OASTH et l'introduction d'une technologie plus coopérative qui prend en compte 


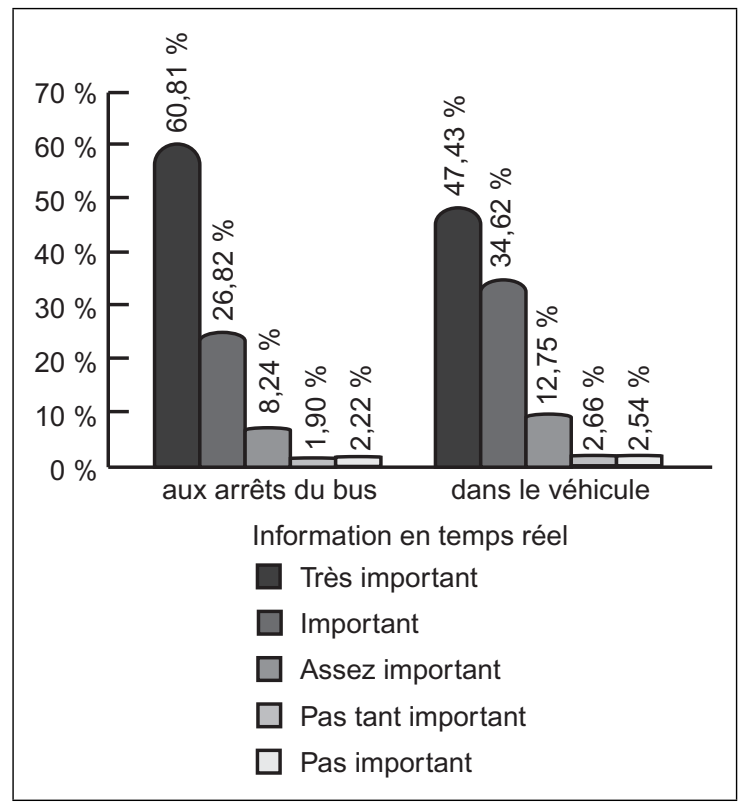

Figure 3 Importance de l'information en temps réel en période d'application complète

les conditions du trafic en temps réel et les retards au départ/à l'arrivée des bus, la perception par les passagers de l'importance de ces technologies est présentée ci-dessous (Figure 3). Les données indiquent que presque $88 \%$ des répondants trouvent ces systèmes d'importance élevée, $2 \%$ croient qu'ils sont assez importants et $4 \%$ ne considèrent pas ces systèmes si nécessaires.

L'évolution dans le temps des valeurs moyennes relatives à l'importance des systèmes d'information en temps réel avant et pendant le voyage est présentée dans la figure 4 . Il est évident qu'après la mise en service du système la manière dont les passagers évaluent ces technologies a changé ; ils les trouvent très importantes et utiles. Cette opinion demeure aujourd'hui, mais a proportion de passagers qui trouvent ces services très importants diminue (10\%-20\%) (Figure 5).

Il semble également que la fourniture d'information en temps réel aux arrêts de bus est un service plus efficace et plus utile pour le passager que la fourniture de l'information à bord et son importance reste élevée dans le temps, avec une petite réduction de $11 \%$. La fourniture de l'information sur l'itinéraire semble être moins importante et il y a également une réduction de $21 \%$ des passagers qui l'ont caractérisée comme « très importante » pendant la période 2007-2010.

Les observations suivantes peuvent être émises : la première étape était une enquête sur les préférences déclarées où la diffusion de l'information, aussi bien aux arrêts et à bord, obtient des pourcentages semblables de réponses « important » et « très important ». C'est probablement l'impact du scepticisme face à la nouveauté

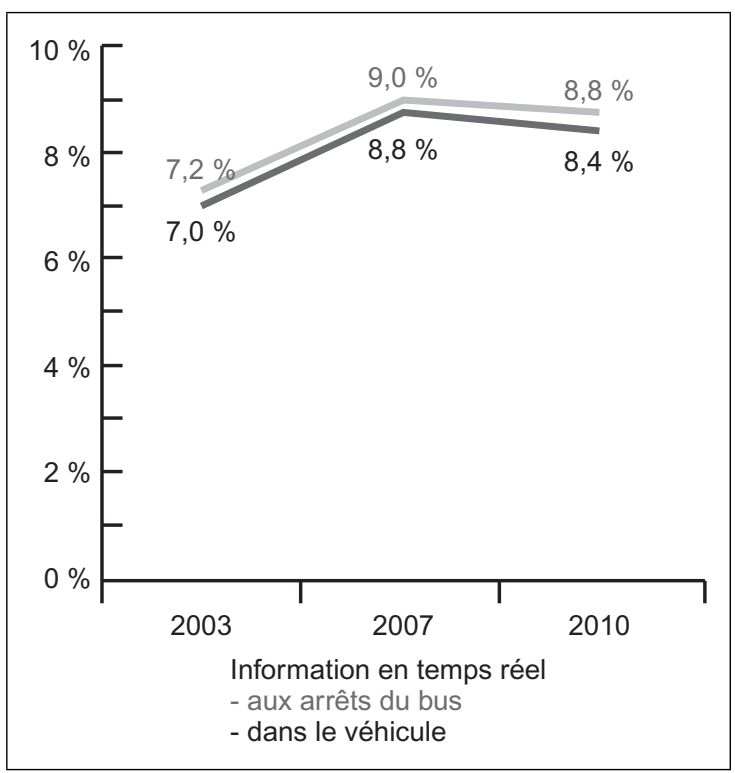

Figure 4 Évolution de l'importance de l'information en temps réel selon le point de vue des passagers

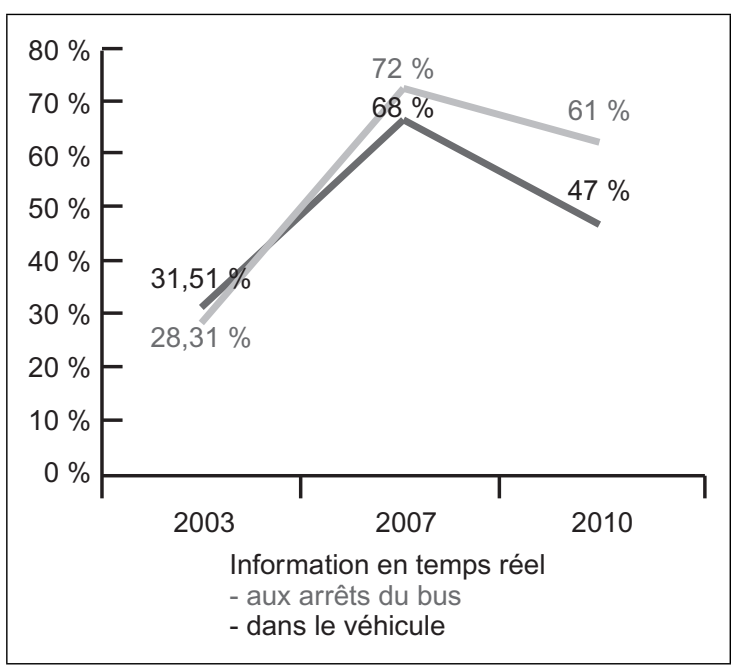

Figure 5 Évaluation de l'information en temps réel jugée « très importante »

ou la difficulté de porter un jugement adéquat pour une application théorique. L'image change dans l'enquête-pilote suivante, basée sur des préférences effectives, où un enthousiasme peut être discerné à travers un pourcentage très élevé de «très important». Ces résultats dénotent un accueil très favorable de la part du public et son appui aux mesures d'amélioration de la qualité de service. Quant à la dernière enquête, elle illustre une réaction plutôt plus « sobre »: il s'agit de déclarations plus sincères vis-à-vis de conditions réelles, étant très probable que les répondants ont déjà une expérience durable des systèmes d'information. 


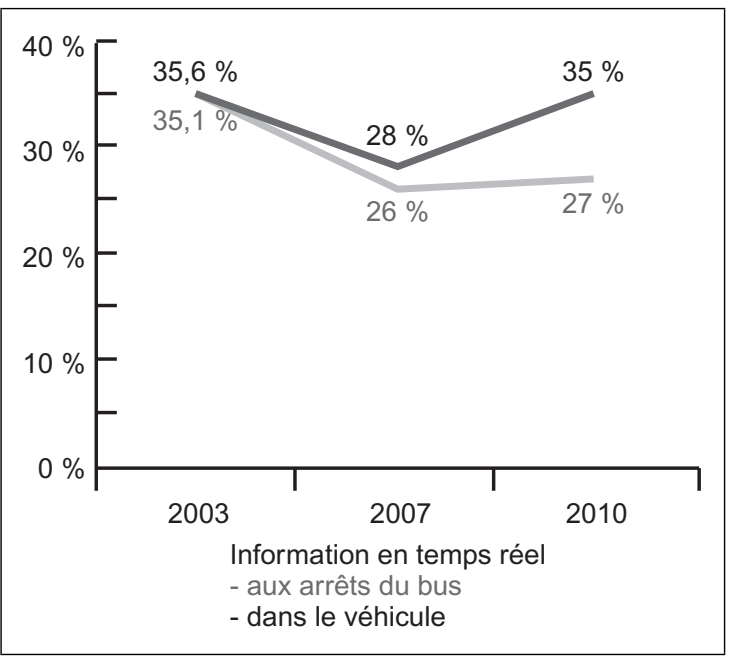

Figure 6 Évaluation de l'information en temps réel jugée « importante »

L'évaluation de l'information en temps réel en tant que composant de la qualité de service des transports publics jugé comme « très important » au fil des ans figure ci-après.

De même, ci-après est indiquée l'évaluation de l'information en temps réel jugée « importante » en tant que composant de la qualité de service des transports publics.

L'analyse des données collectées en trois étapes différentes a permis de dresser les conclusions présentées dans la section suivante.

\section{Conclusions}

L'analyse réalisée sur les données rassemblées de trois enquêtes auprès des utilisateurs réguliers de TP dans la ville de Thessalonique prouve que les systèmes coopératifs d'information en temps réel sont diachroniquement évalués comme importants. Il y a pourtant une grande augmentation (plus de $30 \%$ ) du niveau d'importance entre les périodes avant et après la mise en service et une petite réduction entre la période pilote et celle de la mise en service définitive. L'opinion des passagers semble différer seulement pour le plus haut niveau d'importance, avec une réduction qui atteint $20 \%$ des répondants ayant évalué le système comme «très important » pour la période 2007-2010. Ceci peut être principalement expliqué par le fait que les passagers ont un grand enthousiasme initial face à une nouvelle technologie et, après sa réalisation, ils forment un avis plus éclairé sur son importance.

Un autre résultat de l'analyse est l'évaluation clivée entre les informations fournies aux arrêts de bus et celles affichées à l'intérieur du véhicule. Il semble que les passagers trouvent plus utile et importante l'information aux arrêts, parce qu'elle leur permet encore d'adapter leurs choix. Cette information semble avoir davantage un caractère « coopératif » et peut avoir un impact également sur le volume de passagers du système de transport en commun.

En résumé, on peut conclure que la fourniture d'informations en temps réel a été définitivement jugée importante, ayant été qualifiée constamment comme « importante » ou «très importante » durant les différentes étapes de l'étude. Ce qui peut être facilement observé est que, diachroniquement, la fourniture d'informations est plus appréciée aux arrêts qu'en de route. Cette constatation est conforme à la littérature et confirme aussi la préférence des utilisateurs réguliers [47]. Pour ces derniers, les informations avant le départ sont utiles dans leur vie quotidienne. Les informations à bord des véhicules sont également appréciées, mais ne présentent pas la même utilité pour ce groupe cible, car elles n'offrent plus ni la même liberté de choix, ni une même possibilité de gestion du temps. L'estimation du temps d'attente aux arrêts est couramment mise en avant en tant que principal avantage de l'information en temps réel et peut être cruciale pour la fidélisation des clients. L'information en cours de route pourrait avoir pour eux un caractère confirmatif (bien que cela soit plus apprécié par un utilisateur occasionnel), mais elle serait surtout vraiment utile en cas d'annonce d'incidents et susceptible de minimiser le désagrément ressenti.

En ce qui concerne le potentiel d'augmentation du nombre d'usagers en raison des applications des systèmes d'information, la littérature offre un tableau quelque peu mitigé. Les études de préférences déclarées [47] indiquent qu'une augmentation du nombre d'usagers pourrait être attendue grâce à la présence de systèmes d'annonce en temps réel de l'heure d'arrivée des bus. Ces études indiquent que l'augmentation de l'utilisation serait plutôt le fait des usagers actuels qui augmenteraient leur fréquence d'utilisation plutôt que des non-usagers. Toutefois, le potentiel de ces systèmes à attirer de nouveaux usagers semble aussi non négligeable. La présente étude, à cause de ses propres limitations, ne permet pas de conclure en la matière.

Ceci indique un potentiel pour des recherches futures, qui devraient viser à appréhender plusieurs facteurs qui affectent significativement la tendance des passagers à utiliser plus les transports en commun grâce à une information en temps réel de l'heure d'arrivée. Ces facteurs comprennent notamment le niveau de formation des personnes concernées, l'utilisation actuelle des TP, la distance et la vitesse moyenne des déplacements pendulaires, voire le groupe ethnique. En outre, le choix d'utiliser les TP, au-delà de l'existence d'informations en temps réel, est significativement lié à plusieurs caractéristiques du service des TP, telles que la sécurité perçue et la fréquence des services. Ces résultats 
dénotent que l'information en temps réel peut aider à compenser des lacunes perçues des TP sans devoir investir lourdement pour combler celles-ci [47]. Enfin, les prochains efforts devraient porter sur des enquêtes de préférences effectives pour déterminer les effets réels de l'annonce de l'heure d'arrivée des bus en temps réel, en regardant à la fois les changements comportementaux et psychologiques au niveau du voyageur individuel.

En somme, le fruit de cette recherche était de révéler l'importance des applications STI auprès des utilisateurs et l'appréciation de la satisfaction atteinte par leur mise en service dans le réseau de transports de la région de Thessalonique. Ce travail suggère aussi qu'il y a un lien intéressant entre la présence des STI, l'appréciation du client et l'utilisation éventuelle de TP dont les opérateurs de TP devraient tenir compte et planifier ainsi les interventions et les améliorations de la qualité sur la base d'une liste des priorités. Pourtant, il doit être mentionné que le travail de recherche présenté dans cet article couvre une partie seulement de la recherche nécessaire pour mieux comprendre la relation entre la satisfaction des clients et la qualité de TP en liaison avec les STI et les questions coopératives. Une recherche additionnelle est certainement nécessaire sur l'apparition d'applications coopératives plus avancées dans ce champ d'étude. Un point positif de notre travail est que l'approche méthodologique suivie et les outils utilisés peuvent être repris dans d'autres contextes et cas de TP après une adaptation prudente. Les résultats dépendront cependant toujours des caractéristiques et des besoins spécifiques des clients, de leurs attentes et de leurs préférences.

\section{Recommandations}

La question principale posée par cet article est de savoir si les utilisateurs accordent une importance quelconque aux investissements en systèmes coopératifs avancés. L'intérêt des différents types de services disponibles, tels que l'information en temps réel aux arrêts et à bord, a été également étudié. Nous nous intéressons également à connaitre la relation entre la mise en œuvre de ces systèmes et le niveau de service des TP, en vue d'attirer plus de voyageurs. Pour ces questions de recherche, nous avons utilisé et compilé les données de trois enquêtes différentes avec l'objectif d'étudier la possibilité de changements comportementaux induits par l'information aux voyageurs.

L'affichage d'informations en temps réel aux arrêts augmente l'attrait des TP en général. Ceci contribue à augmenter le nombre de clients, mais il est difficile de quantifier cette augmentation car les résultats mesurés résultent souvent de combinaisons avec d'autres facteurs telles que des campagnes de marketing ou la mise en place de nouveaux itinéraires. Plusieurs études ont montré que les avantages immédiats augmentent avec le temps, les voyageurs appréciant d'autant plus l'information qu'ils l'utilisent. Ce travail confirme cette appréciation en illustrant l'importance constante que les utilisateurs accordent à ces systèmes.

De nombreuses études montrent que l'affichage aux arrêts réduit l'incertitude et le temps d'attente ressentis et augmente la sécurité perçue. Plus l'affichage est avancé, plus grand est le potentiel de planification par l'usager grâce aux options qui sont à sa disposition (en particulier en cas de perturbation du service). Comme l'indique la littérature sur la psychologie des attentes, la satisfaction globale s'élève et des changements d'autres réactions affectives (dans ce cas, le niveau d'anxiété et le sentiment de sécurité) contribuent à élever le niveau de satisfaction globale [53]. Nos résultats sont conformes avec les conclusions d'études précédentes selon lesquelles les usagers actuels apprécient l'information en temps réel, l'utilisent et se sentent plus à l'aise lorsqu'ils en disposent [37].

Dans ce contexte, notre focalisation sur la clientèle actuelle est pertinente. Mettre l'accent sur les utilisateurs fréquents, peut être un bon point de départ pour des actions ultérieures plus ciblées. Malgré la tendance générale parmi la population étudiée d'indiquer qu'ils utiliseraient plus les transports en commun en présence de ce type d'informations (aux arrêts ou en cours de route), nos résultats ne peuvent que modestement informer et orienter les priorités de l'opérateur. Les priorités d'investissement pourraient impliquer des services plus avancés aux arrêts visant à renforcer les relations avec les clients actuels. La tendance constatée d'utiliser plus les transports en commun devrait être étudiée soigneusement en prenant en compte plusieurs paramètres - tels que le sexe, le niveau d'éducation, la valeur du temps des utilisateurs, etc. - ayant comme base les préférences effectives de ces derniers sur une période de temps logiquement choisie après la mise en œuvre de systèmes coopératifs. La constatation et la mesure de l'ampleur des nombreux effets nécessite du temps du fait que l'adaptation du comportement humain se produit avec une certaine hystérèse. Beckmann indique qu'une enquête d'évaluation définitive ne devrait être posée que 3-5 ans après la mise en œuvre [54].

Les résultats indiquent que les informations en temps réel peuvent également aider à alléger les carences du service, telles que la sécurité ou le manque de fréquence qu'affrontent des TP actuels, et ceci sans investissements coûteux. En d'autres mots, les systèmes d'information en temps réel sont une alternative, mais aussi un moyen efficace pour répondre à plusieurs préoccupations liées aux TP lorsque ces questions sont trop difficiles ou coûteuses pour y apporter une solution directe. Ces résultats ont plusieurs conséquences importantes sur la politique et sur 
la conception des services et plus d'efforts devraient y être consacrés, en particulier si des systèmes coopératifs plus compliqués devaient être déployés. Fournir une information fiable est un composant essentiel et exigeant du service des $\mathrm{TP}$, parce que le voyageur peut immédiatement comparer l'exactitude de l'information avec la réalité. S'il est déçu par la qualité des informations en temps réel, regagner sa confiance est difficile. Dans un contexte de TP, il est souhaitable de se concentrer davantage sur les clients et leurs besoins que sur les défis techniques. Le développement devrait toujours commencer et se concrétiser en ayant le client en point de mire.

Le faible volume des recherches empiriques menées à ce jour, et les disparités de leurs conclusions, démontrent le besoin d'études ultérieures. L'évaluation de l'information aux voyageurs des TP doit comprendre les réactions de l'utilisateur, ses perceptions et son attitude face aux systèmes. Idéalement, ceci devrait être segmenté selon plusieurs groupes cibles. Le problème est que de nombreuses études utilisent des informations obtenue des utilisateurs de manière différente chacune. Des observations du comportement réel des passagers sont rares dans ce genre d'études. En outre, il est important d'associer les préférences constatées et celles déclarées avec des observations directes selon des méthodologies d'étude du comportement afin d'obtenir des résultats valides.

Plus largement, l'échange d'outils, de méthodologies, d'expériences et de résultats d'essai grâce à des initiatives dans le monde entier est une aide à la décisions quant au déploiement et quant à une standardisation commune minimale ; ce qui est vital pour concevoir des systèmes efficaces pour chaque région.

En Grèce, une analyse des besoins et des pistes de recherche plus systématique et des actions éventuelles en matière de systèmes coopératifs devrait être entreprise en tant qu'entrée stratégique pour la planification des investissements à grande échelle dans le domaine de TP au niveau national. Les opérateurs TP peuvent être les leviers pour de telles initiatives, mais les autorités locales devraient se rendre compte du potentiel des systèmes coopératifs dans le contexte grec et les rejoindre. Par ailleurs, étant un État-membre de l'Union Européenne et compte tenu de la réalité économique, il y a besoin d'adopter une vision, une architecture et des normes européennes communes. Des travaux sont en cours à ce sujet et la Grèce doit profiter de l'expérience des autres afin d'élaborer sa propre stratégie en matière de STI. Les échanges de bonnes pratiques et d'évaluation des STI entre les villes européennes permettront d'acquérir une expertise en la matière [55].

Une stratégie de systèmes de transport intelligents porte sur l'investissement dans des solutions novatrices afin de rendre l'infrastructure de transport plus sûre et efficace.
En mettant l'accent sur les principaux besoins des usagers, la stratégie contribue à produire des résultats positifs pour la population. C'est un outil pour les exploitants de TP afin d'augmenter le nombre de leurs passagers, et pour les autorités afin de mieux gérer l'encombrement sur les routes. Les opérateurs de TP et les autorités centrales et locales doivent collaborer avec des équipes de recherche et l'industrie afin d'élaborer des solutions innovantes et, conjointement, les mettre en œuvre efficacement et ériger une infrastructure de transport compétente et intégrée pour l'avenir. Pour sa part, le gouvernement devrait tenter de minimiser les risques inhérents à la mise au point des produits, simplifier et coordonner l'accès à l'information entre divers niveaux et organismes, afin de réduire le nombre et la complexité des négociations auxquelles la nouvelle industrie des services d'information aux voyageurs est confrontée.

Enfin et surtout, des utilisateurs satisfaits par les STI peuvent vraiment amplifier leur déploiement, impliquant les systèmes coopératifs. Atteindre ce but exige de développer des applications d'importance pour les objectifs politiques de l'autorité locale et des besoins de l'utilisateur, en particulier quant aux transports en commun et ceux des utilisateurs non-motorisés. Une évaluation appropriée des avantages, particulièrement au niveau urbain, devrait être assurée. Des efforts devraient être faits afin de développer des scénarios concrets de déploiement pour des autorités locales, montrant (entre autres choses) comment passer d'une manière rentable des systèmes existants aux systèmes coopératifs. Développer des bons business plans pour les autorités locales et la communauté ferait du déploiement des systèmes coopératifs un but commun.

\section{Références}

1. Lyons G (2001) From Advanced towards effective traveler information systems. The leading edge, ed. Hensher D., Pergamon, International Association for Travel Behaviour Research, pp. 813-825.

2. Chorus C, Arentze Timmermans H (2007) Information impact on quality of multimodal travel choices: conceptualizations and empirical analyses. Transportation, Springer.

3. Commission of the European Communities (2001) European transport policy for 2020: Time to decide. Luxembourg.

4. Department for Transport (2004) The future of transport: a network for 2030. London, UK.

5. Dziekan K, Vermeulen A (2004) The added value of real-time information and effects to customer behaviour-A before-after study of the implementation of a new real-time information system. Project Report. Stockholm/The Hague: KTH/ HTM.

6. Casey R, Labell L, Prensky S, Schweiger C (1991) Advanced public transportation systems: The state of the art. USDOT/FTA Report No. DT-VNTSC-UMTA- 91-2.

7. Drouet D, Sellier D (2000) La recherche aux États-Unis dans le domaine des transports terrestres. PREDIT. 
8. Zhang G, Li M, Wang J (2007) Application of the Advanced Public Transport System in Cities of China and the Prospect of Its Future Development. Journal of Transportation Systems engineering and information technology 7(5): 24-30.

9. Yeung T (2004) Editorial to "Using IT tools to improve service" Public Transportation International 53(6): 2-3.

10. Dziekan K, Kottenhoff K (2007) Dynamic at-stop real-time information displays for public transport: effects on customers. Transportation Research Part A 41: 489-501.

11. Politis I, Papaioannou P, Basbas S, Dimitriadis N (2010) Evaluation of a bus passenger information system from the users' point of view in the city of Thessaloniki, Greece. Research in Transportation Economics 29: 249-255.

12. Daskalakis N, Stathopoulos A (2008) User's perceptive evaluation of bus arrival time deviations, in stochastic networks. Journal of Public Transportation 11(4). Disponible sur : www.nctr.usf.edu/jpt/pdf/JPT11-4Daskalakis.pdf.

13. Federal Transit Administration (FTA), United States Department of Transportation (U.S. DOT), (2003) Guidance for Developing and Deploying Real-time Traveller Information for Transit. Intelligent Transportation Systems (ITS) Joint Program Office (JPO), U.S. DOTFTA-OH-26-7017-2003.1, April.

14. United States Department of Transportation (2002) ITS standards program.

15. United States Department of Transportation (2006) Final report on real time bus arrival information systems return on investment study.

16. Papaioannou P, Mikiki F, Morfoulaki M (2008) Exploring ITS impact on customers satisfaction and travel behaviour mechanism, Proceeding of the 10th International Conference on Application of Advanced Technologies in Transportation, Athens, Greece, May, 27-31.

17. Lappin J (2002) What have we learned about advanced traveller information systems and customer satisfaction? John A. Volpe National Transportation System Centre. Disponible sur : http://ntl.bts.gov/lib/jpodocs/repts_te/13320.pdf.

18. SAIC, USDoT (2003) Oregon Statewide Transit Trip Planning Evaluation Plan. Disponible sur : http://www. itsdocs.fhwa.dot.gov/JPODOCS/REPTS_TE/13835_files/13835. pdf.

19. Science Applications International Corporation (2003) Transit tracker information displays, oregon regional intelligent transportation systems. Integration Program, Final Phase III Report, USA

20. Geerlings H, Klementschitz R, Mulley C (2006) Development of a methodology for benchmarking public transportation organizations: a practical tool based on an industry sound methodology. Journal of Cleaner Production 14: 113-123.

21. Benjamin J (2006) A Study of the Impact of APTS on Service Quality Perceptions of Elderly and Disabled Riders. Journal of Public Transportation 9(1): 53-70.

22. Cham L, Darido G, Jackson D, Laver R, Schneck D (2006) Real-time Bus Arrival Information Systems Return-on-Investment Study. Final Report, US Department of Transportation.

23. Polère C (2011) Robotique et mobilité, La perspective d'une cité des transports automatisés est-elle crédible? synthèse pour Grand Lyon, communauté urbaine Direction de la Prospective et du Dialogue Public, Mars.

24. POLIS network (2011) Position paper on ITS. 6p. Disponible sur : www.polis-online.org.

25. ERTICO, (2012) disponible sur : http://www.ertico.com/ cooperativemobility/

26. Konstantinopoulou L (2010), Non-technical barriers to deployment of cooperative systems. Transport Research Arena, Brussels.
27. Morfoulaki M, Papaioannou P (2006) Measuring Customer Satisfaction Index: the Survey of Thessaloniki-Greece. Paper presented at the 85th Annual Meeting of the Transportation Research Board, Washington DC, January.

28. Friman M, Garling T (2001) Satisfaction with public transport related to service performance, The leading edge. ed. Hensher D., Pergamon, International Association for Travel Behaviour Research, pp. 845-854.

29. Hunte-Zaworski K (2004) Transit Capacity and Quality of Service Manual (2nd Edition), Transportation Research Board, National Academy Press, Washington DC.

30. EU-QUATTRO project consortium, (1998) RTD Programme QUATRO-Quality approach in tendering urban public transport operations, Final report.

31. Morpace International, Inc., Cambridge Systematics Inc., (1997) A Handbook for Measuring Customer Satisfaction and Service Quality. Transportation Research Board, TCRP Report 47, National Academy Press, Washington DC.

32. EU-EQUIP project consortium, (2000) RTD Programme EQUIP-Extending the Quality of Public Transport, Final report and annex (practical handbook).

33. University of Southampton, Transportation Research Group, (2006) EU- MIRACLES project Final Deliverable D4.2 : Report on Evaluation Results Version 4.0.

34. Dziekan K (2004) Customer perceptions and behavioural responses to IT based public transport information-Literature review and what the experts say. (No. TRITA-INFRA 04-012), Stockholm: Royal Institute of Technology Stockholm.

35. Papaioannou P, Basbas S, Vougioukas M (1996) The use of Stated Preference Technique in Evaluating a Passenger Information System : The EUROBUS/POPINS/THEPIS experience. 2-PTRC-ETF Proceedings, Volume P 405, Brunel, UK.

36. Lappin J, Bottom J (2001) Understanding and Predicting Traveler Response to Information: A Literature Review. Washington, DC, Office of the Metropolitan Planning, Federal Highway Administration.

37. Turnbull K, Pratt R (2003) "Traveler Response to Transportation System Changes". Chapter 11 of Transit Information and Promotion, Washington, D.C ; Transportation Research Board/National Academy Press Schweiger.

38. TRB 2003, Real-Time Bus Arrival Information Systems, A Synthesis of Transit Practice, TCRP SYNTHESIS 48.

39. Abdel-Aty M (2001) "Using ordered probit modeling to study the effect of ATIS on transit ridership". In Transportation research, Part C, Emerging technologies 9C(4).

40. Stöveken P (2000) Field study of real time passenger information via mobile phone. 7th World Congress on ITS, Turin/ Italy.

41. Stöveken P, Arndt K (2001) "Fahrgastinformation über Handy erhöht Kundenzufriedenheit. Ergebnisse eines Feldversuches in Hessen." Der Nahverkehr 19(10): 18ff.

42. Kronborg P, Lindkvist A, Schelin E (2002) Fungera transportinformatik $i$ praktiken? Fallstudier $i$ syfte att undvika misstag $i$ framtiden. Stockholm, TFK (Institut för transportforskning).

43. Dziekan K, Vermeulen A (2006) Psychological Effects of and Design Preferences for Real-Time Information Displays. Journal of Public Transportation 9(1): 71-89.

44. Molin E, Timmermans H (2006) "Traveler Expectations and Willingness-to-pay for Web-enabled Public Transport Information Services" In Transportation Research Part C 14: $57-67$.

45. National Center for Transit Research, Center for Urban Transportation Research, University of South Florida (2005). Enhancing the Rider Experience : The Impact of Real-Time Information On Transit Ridership, Progress Report, July. 
46. Chorus C, Molin E, Van Wee B, Arentze T, Timmermans H (2006) "Responses to Transit Information among Car-drivers: Regret-based Models and Simulations". Transportation Planning \& Technology 29(4): 249-271.

47. Tang L, Thakuriah P (2006) An Analysis of Behavioral Responses to Real-Time Transit Information Systems. Presented in the Association of Collegiate Schools of Planning annual meeting. Fort Worth.

48. Zhang F, Shen Q, Clifton K (2008) An Examination of Traveler Responses to Real-time Bus Arrival Information Using Panel Data, Proceedings of TRB 2008 Annual Meeting.

49. Denco Trademco, Infodim Aggelidis, Truth SDG, Atkins WS (2000) Organization for the Master Plan and Environmental Protection of Thessaloniki, General Transport Study (GTS) of Thessaloniki Metropolitan Area, Thessaloniki, Greece.

50. Urban Transport Organization of Thessaloniki (OASTH), (2009) Retrieved June 26, 2009, website, www.oasth.gr.
51. Ben-Akiva M, Lerman S (1985) Discrete choice analysis: Theory and application to travel demand. Cambridge, Massachusetts, USA: The MIT Press.

52. Morfoulaki M (2004) Research in the quality measures and the role of benchmarking in the public transport system of Thessaloniki, $\mathrm{PhD}$ Thesis, Aristotle University of Thessaloniki, Thessaloniki, Greece.

53. Taylor S, Fullerton G (2000) Waiting for service: perceptions management of the wait experience. In T.A. Swarz, D. Iacobucci, Services Marketing and Management, Sage publications. pp. 171-190.

54. Beckmann K.J (2003) "Mobilität in Ballungsräumen - Entwicklungstendenzen, Anforderungen, Beiträge der Leitprojekte. Verkehrszeichen 1: 4-8.

55. CONDUITS project consortium, (2011) Project newsletter 4, disponible sur : www.conduits.eu. 